\title{
PEDIATRIC IMAGING
}

\section{Iranion foumat of}

\section{Diagnostic Accuracy of Ultrasound in Determining the Cause of Bilious Vomiting in Neonates}

\author{
Mehdi Alehossein ${ }^{1,2}$, Siamak Abdi ${ }^{3}$, Mohammad Pourgholami ${ }^{2}$, Mohsen Naseri ${ }^{4}$, Payman \\ Salamati ${ }^{1^{*}}$
}

${ }^{1}$ Advanced Diagnostic and Interventional Radiology Research Center (ADIR), Medical Imaging Center, Imam Khomeini Hospital, Tehran University of Medical Sciences, Tehran, Iran

2 Department of Radiology, Bahrami Hospital, Tehran University of Medical Sciences, Tehran, Iran

${ }^{3}$ Department of Neurology, Shariati Hospital, Tehran University of Medical Sciences, Tehran, Iran

${ }^{4}$ Department of Pediatrics, Bahrami Hospital, Tehran University of Medical Sciences, Tehran, Iran

* Corresponding author: Payman Salamati, Advanced Diagnostic and Interventional Radiology Research Center (ADIR), Medical Imaging Center, Imam Khomeini Complex Hospital, Keshavarz Blvd., Tehran, Iran. Tel.: +98-2166581579, Fax: +98-2166581560, E-mail: psalamati@tums.ac.ir

\section{A B S T R A C T}

Background: Plain radiography and contrast radiologic studies are traditionally the main options in evaluating neonates presenting with bilious vomiting. While ultrasonography (US) is more available, its diagnostic accuracy is in question.

Objectives: The purpose of this study is to determine the diagnostic accuracy of US in evaluating these patients with bilious vomiting.

Patients and Methods: All neonates with bilious vomiting or bilious nasogastric tube drainage presented to a children's hospital in a 1.5year period were included. US were performed in all patients. The results were compared with clinical and radiological data and the final diagnosis. We used chi-square and Fisher's exact tests for analysis.

Results: The cause of bilious vomiting for 18 of the 23 included patients was surgical. All patients labeled as surgical candidates by US ended in surgery [positive predictive value (PPV) $=100 \%$, while only $50 \%$ of the patients with inconclusive US were operated [negative predictive value $(\mathrm{NPV})=50 \%$, Confidence Interval (CI) 95\%: 29\%-71\%]. The sensitivity and specificity of US in diagnosing intestinal atresia ( $\mathrm{n}=9)$ was $89 \%$ [CI 95\%: $(68 \%-100 \%)]$ and $100 \%$. In cases with malrotation $(n=4)$ and midgut volvulus $(n=2)$, sonographic diagnosis was in concordance with final surgical diagnosis.

Conclusion: This study suggested that in cases in which US makes a certain diagnosis, its accuracy eliminates the need for further diagnostic tests, but if it is inconclusive, further radiological contrast studies should be tried to make the final diagnosis.

Keywords: Ultrasonography; Vomiting; Infant, Newborn; Bilious

Copyright () 2012, Tehran University of Medical Sciences and Iranian Society of Radiology. Published by Kowsar Corp. All rights reserved.

Article type: Research Article; Received: 12 Jul 2011, Revised: 04 Sep 2012, Accepted: 08 Sep 2012; DOI:10.5812/iranjradiol.8465

Implication for health policy/practice/research/medical education:

Performing US in all neonates with bilious vomiting can depict surgical cases well if there are positive findings in the sonogram. In such cases, US can replace UGI series, resulting in decrease in the radiation dose, cost and time.

Please cite this paper as:

Alehossein M, Abdi S, Pourgholami M, Naseri M, Salamati P. Diagnostic Accuracy of Ultrasound in Determining the Cause of Bilious Vomiting in Neonates. Iran J Radiol. 2012; 9 (4):190-4. DOI: 10.5812/iranjradiol.8465

Copyright (C) 2012, Tehran University of Medical Sciences and Iranian Society of Radiology; Published by Kowsar Corp.

This is an Open Access article distributed under the terms of the Creative Commons Attribution License (http://creativecommons.org/licenses/by/3.0), which permits unrestricted use, distribution, and reproduction in any medium, provided the original work is properly cited. 


\section{Background}

Bile vomiting in a neonate has traditionally been the cardinal sign of intestinal obstruction. In the absence of clinical signs or a plain abdominal film allowing a positive diagnosis, an upper gastrointestinal (UGI) contrast study is recommended to specifically exclude intestinal malrotation and volvulus $(1,2)$ which are the most emergent differential diagnoses. There are limitations for $\mathrm{X}$-ray studies in neonates including radiation hazards, physical trauma such as the risk of hypothermia, difficult positioning and being time consuming (3). Meanwhile, there are studies which show that intestinal obstruction is not the cause of bilious vomiting in one half to two third of infants and most of these infants recover with no further sequel $(4,5)$. Performing routine UGI series for most of these neonates may be superfluous.

Several reports of the high accuracy of ultrasonography (US) in the diagnosis of midgut volvulus (6-8) made us interested to find whether using US as a safer and faster method may decrease standard UGI series usage in diagnosing the cause of bilious vomiting in neonates.

\section{Objectives}

The main aim of this study is to determine the diagnostic accuracy of US in evaluating patients with bilious vomiting.

\section{Patients and Methods}

\subsection{Participants}

Neonates with the history of bilious vomiting or bilious nasogastric drainage referred to Bahrami Children's Hospital in Tehran were evaluated by ultrasonography. Neonates with definite diagnosis made by plain abdominal radiography were excluded. Neonates with a low position of nasogastric tube in the duodenum were not included in this prospective study.

\subsection{Test Methods}

Abdominal US were performed with a real time US unit (Siemens G50 with 7.5 convex and 10MHz linear transducers). Routine gray scale US was done by a pediatric radiologist. From the technical point of view, the baby was first located in the right lateral decubitus to displace gastric secretions toward the antropyloric portion and duodenum. In some stable cases, few milliliters of water were instilled via the nasogastric (NG) tube. We first characterized the antropyloric portion. The duodenal bulb was visualized as an arrowhead. Then we followed the descending portion of the duodenum lateral to the pancreatic head, the third horizontal portion (between the aorta and the superior mesenteric artery) and finally the fourth portion (cephalad and to the left). More distal bowel loops were followed by keeping the graded compression technique. Small and large bowels could be differentiated according to anatomic landmarks and their locations. The level of bowel obstruction may be determined by change in the caliber of the bowel loop or direct visualization of pathology (Figure 1). We reported details with clarification anatomy of the duodenum, distention or collapse of bowel loops, free fluid or other abnormalities. Color Doppler US was also conducted with attention to vascularization of the wall of the gastrointestinal tract, superior mesenteric vein/superior mesenteric artery (SMV/SMA) orientation and whirlpool sign (Figure 2).The whirlpool sign was recognized as a representative for midgut volvulus on transverse sonograms of the upper abdomen. The direction of the whirlpool was determined as clockwise or counterclockwise viewed from below the patient. When the transducer was moved in a craniocaudal direction on the abdomen, the whirlpool on the monitor whirled clockwise or counterclockwise according to the direction of the volvulus and the direction of the transducer motion. Surgical cases were implied in the sonographic report as well as inconclusive cases. Routine clinical care remained unchanged during the study period and some patients had UGI series too. A chart review was performed to document clinical information including the outcome that was obtained in the operating room or follow up in the neonatal ward.

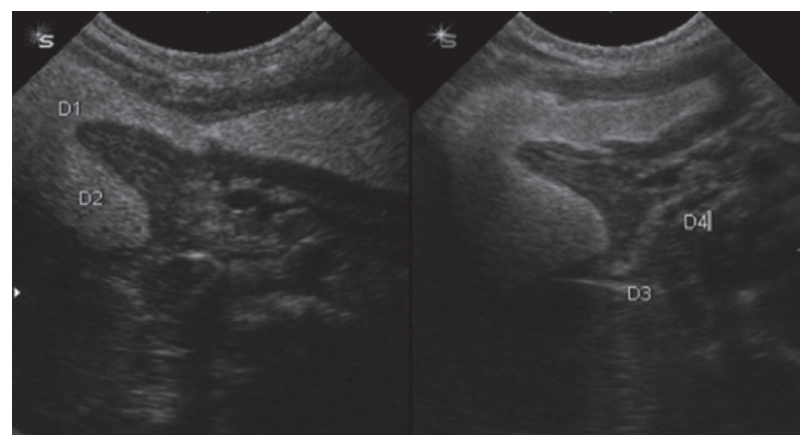

Figure 1. A 21-day neonate with bilious vomiting

Distended bulb (D1) and descending (D2) portions of the duodenum while horizontal (D3) and ascending (D4) portions of the duodenum are collapsed. Partial obstruction due to the web is nicely demonstrated.

\subsection{Statistical Analysis}

We used the SPSS software (version 16) for all data analyses. We analyzed data using Chi-Square and Fisher's exact tests and statistical type one error was considered lower than $5 \%$.

\section{Results}

\subsection{Participants}

The study was performed from December 1,2007 to May 1,2009. Twenty-three neonates (13 boys, 10 girls) considering inclusion criteria were identified. Their median age 
was 7.8 days (range, 1 to 27). Their median weight at birth and admission was 2922 and 2865 grams, respectively.

\subsection{Test Results}

Table 1 summarizes the findings of this study. Eighteen neonates (78\%) had surgical pathology; jejunal atresia (n $=5)$, duodenal obstruction $(n=4)$, malrotation $(n=4)$, Hirschprung disease $(n=2)$ and miscellaneous $(n=4)$. One neonate had both malrotation and jejunal atresia according to the surgical report. Sensitivity and specificity of US in diagnosing intestinal atresia $(n=9)$ was $89 \%$ (CI 95\%: 68\% - 100\%) and 100\%. In cases with malrotation $(\mathrm{n}=4)$ and midgut volvulus $(\mathrm{n}=2)$, sonographic diagnosis was in concordance with the final surgical diagnosis. Miscellaneous cases consisted of esophageal atresia with tracheoesophageal fistula bypassing gastric vomitus ( $\mathrm{n}=$ 1), hypertrophic pyloric stenosis with initial impression of bilious vomiting $(n=1)$, Hirschprung disease $(n=1)$ and adhesion band $(n=1)$. Five neonates $(22 \%)$ were cured by medical management alone; three neonatal sepsis cases and two meconium plug disease cases. Seventeen neonates had plain abdominal radiography; normal $(\mathrm{n}=$ $5)$, gasless abdomen $(n=4)$, generalized intestinal distention $(n=3)$ and focal distention $(n=5)$.

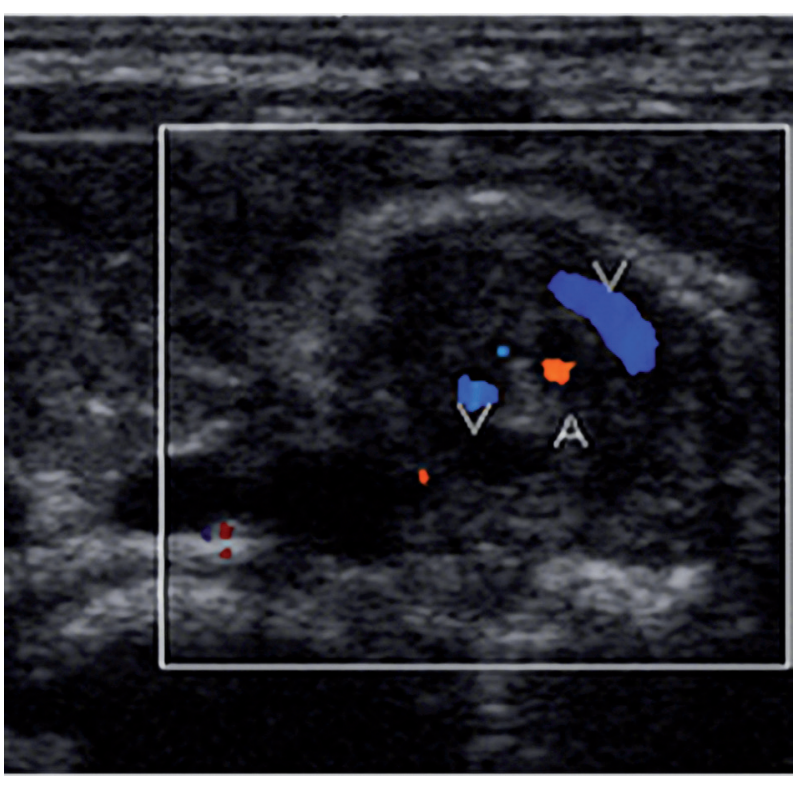

Figure 2. A 5-day male neonate with bilious vomiting

Transverse color Doppler sonogram of the upper abdomen shows a whirlpool sign with a clockwise direction. Capital letter A indicates SMA and V indicate SMV.

Three patients had UGI series; two had malrotation and one was normal. In 12 cases (52.2\%), there was concordance between sonographic diagnosis and final diagnosis. In 10 cases (43/5\%), there was inconclusive sonographic diagnosis and there was one patient with more than one diagnosis at operation.
Superior mesenteric artery (SMA) - superior mesenteric vein (SMV) displacement was seen in all patients with malrotation. While the final diagnosis for both patients with SMA/SMV inversion was volvulus, it was the final diagnosis only for $33 \%$ of the patients with ventral SMV. All patients with volvulus had the whirlpool sign, although there was one false positive. All patients labeled as surgical candidates by US ended in surgery (PPV $=100 \%$ ), while only $50 \%$ of the patients with inconclusive US were operated $(\mathrm{NPV}=50 \%$, CI 95\%: $29 \%-71 \%)$.

\section{Discussion}

Standard teaching in pediatric surgery mentions that bile vomiting in a neonate indicates intestinal obstruction until proven otherwise (1). There are studies which show that bilious vomiting in the neonatal period is not invariably associated with intestinal obstruction and the rate of intestinal obstruction may be as low as $38 \%$ $(4,5)$. Surgical intervention is necessary in $30 \%$ to $40 \%$ of neonates with bile vomiting (9). However, in our study, $78 \%$ of neonates with bilious vomiting required surgical intervention. As a referral pediatric surgical center, our hospital frequently admits patients with previous hospitalization which may account for the higher operation rate reported in the present study.

Although UGI series are considered as the modality of choice in the diagnosis of malrotation, there are shortcomings in the diagnosis or exclusion of malrotation with an up to $30 \%$ false positive rate, the risk of radiation exposure and the time consuming characteristic of the procedure. UGI series were performed only in three of our cases as routine clinical care and they remained unchanged during the study period, but color Doppler sonography was performed in all of them, referring to the policy of Schimanki et al. as the initial imaging study in children suspected of having midgut volvulus. Normally, the SMV is on the right side of the artery. In malrotation, the mesenteric vein is on the left side of the artery. However, this inversion is neither specific nor sensitive enough. A normal sonogram does not exclude malrotation (10). There are also other data which show that sonography is a good screening tool that effectively rules out malrotation at risk for volvulus (7). We had four cases (17.3\%) of malrotation and three cases with the sonographic diagnosis of midgut volvulus of which finally two were surgically confirmed as midgut volvulus. The other case was as apparent over diagnosis of midgut volvulus by US that was detected as malrotation and jejunal atresia in surgery. This association was noted in literature as $28 \%$ of duodenal atresia and $19 \%$ of jejunoileal atresia had malrotation (2). The whirlpool sign in cases of volvulus refers to the sonographic appearance of the SMV, along with the bowel and mesentery wrapping in a clockwise mode around the SMA (11). Although it is accurate in the diagnosis of midgut volvulus, there are some case reports of the whirlpool sign being present in the absence of volvu- 
lus $(12,13)$. However, our case may be due to spontaneous devolvulation, considering that the simultaneous UGI series performed on this case was compatible with malrotation and volvulus (corkscrew sign). The counterclockwise whirlpool-like pattern of SMA and SMV was noted in an- other case with the final diagnosis of imperforated anus and no malrotation or volvulus of the midgut. There was one interesting case of esophageal atresia with tracheoesophageal fistula which bypassed the gastric contents to the proximal esophagus.

\begin{tabular}{|c|c|c|c|c|c|c|}
\hline Age, $y$ & Gender & Weight & $\begin{array}{l}\text { SMA-SMV } \\
\text { Position }\end{array}$ & US Diagnosis & Management & Final Diagnosis \\
\hline 6 & Male & 2650 & Normal & JA & Surgical & JA \\
\hline 7 & Male & 2350 & $\mathrm{AP}$ & JA & Surgical & JA \\
\hline 23 & Male & 2150 & $\mathrm{AP}$ & Malrotation & Surgical & Malrotation \\
\hline 12 & Male & 2000 & Normal & NSF & Medical & Meconium plug \\
\hline 11 & Male & 2900 & AP & NSF & Surgical & Adhesion bands \\
\hline 3 & Male & 4250 & Normal & NSF & Medical & Meconium plug \\
\hline 21 & Female & 2450 & Normal & Duodenal web & Surgical & Duodenal web \\
\hline 3 & Male & 2600 & Normal & NSF & Surgical & HD \\
\hline 2 & Female & 2700 & Normal & NSF & Surgical & EA \\
\hline 8 & Female & 2650 & Reverse & MGV & Surgical & MGV \\
\hline 4 & Male & 3400 & Normal & NSF & Surgical & ARM \\
\hline 11 & Female & 3350 & Normal & $\begin{array}{l}\text { Duodenal } \\
\text { obstruction }\end{array}$ & Surgical & DA \\
\hline $\mathbf{1}$ & Female & 3150 & Normal & JA & Surgical & JA \\
\hline 2 & Female & 2400 & Normal & JA & Surgical & JA \\
\hline 27 & Male & 4400 & Normal & HPS & Surgical & HPS \\
\hline $\mathbf{1}$ & Male & 2750 & Normal & NSF & Surgical & HD \\
\hline 2 & Female & 2200 & Normal & $\begin{array}{l}\text { Duodenal } \\
\text { obstruction }\end{array}$ & Surgical & Duodenal web \\
\hline 11 & Female & 2380 & $\mathrm{AP}$ & NSF & Medical & Sepsis \\
\hline 5 & Male & 3550 & Reverse & MGV & Surgical & MGV \\
\hline 5 & Male & 2250 & $\mathrm{AP}$ & MGV & Surgical & JA and MGV \\
\hline 3 & Male & 3100 & $\mathrm{AP}$ & $\begin{array}{l}\text { Duodenal } \\
\text { obstruction }\end{array}$ & Surgical & DA \\
\hline 3 & Female & 2780 & Normal & NSF & Medical & Sepsis \\
\hline 12 & Female & 3900 & Normal & NSF & Medical & Sepsis \\
\hline
\end{tabular}

Abbreviations: AP; anteroposterior, ARM; anorectal malformation, DA; duodenal Atresia, EA; esophageal atresia, HD; Hirschprung's disease, HPS; hypertrophic pyloric stenosis, JA; jejunal atresia, MGV; midgut volvulus, NSF; nonspecific findings, SMA; superior mesenteric Artery, SMV; superior mesenteric vein

Another unusual case with hypertrophic pyloric stenosis was noted with initial impression of bilious vomiting. Contrast enema was performed when there was a high probability of lower obstruction in order to search for meconium ileus, ileal atresia, meconium plug syndrome, Hirschsprung's disease and colonic atresia.

In conclusion, performing US in all neonates with bilious vomiting can depict surgical cases well if there are positive findings in the sonogram. In such cases, US can replace UGI series, resulting in decrease in the radiation dose, cost and time. The latter is very important in midgut volvulus which may be life threatening if not promptly operated.

\section{Acknowledgments}

None declared.

\section{Authors' Contribution}

The manuscript was the result of Dr. Siamak Abdi's thesis. Dr. Mehdi Alehossein was the supervising professor and Dr. Payman Salamati was the advising professor of the the- 
sis. Dr. Mohammad Pourgholami and Dr. Mohsen Naseri helped in reviewing the literature and manuscript writing.

\section{Financial Disclosure}

None declared.

\section{Funding/Support}

None declared.

\section{References}

1. Walker GM, Raine PA. Bilious vomiting in the newborn: how often is further investigation undertaken? J Pediatr Surg. 2007;42(4):714-6.

2. Strouse PJ. Disorders of intestinal rotation and fixation ("malrotation"). Pediatr Radiol. 2004;34(11):837-51.

3. Kirks DR, Griscom NT. Practical Pediatric Imaging: Diagnostic Radiology of Infants and Children. 1997.

4. Godbole P, Stringe MD. Bilious vomiting in the newborn: How often is it pathologic? J Pediatr Surg. 2002;6:909-11.

5. Lilien LD, Srinivasan G, Pyati SP, Yeh TF, Pildes RS. Green vom- iting in the first 72 hours in normal infants. Am J Dis Child. 1986;140(7):662-4.

6. Shimanuki Y, Aihara T, Takano H, Moritani T, Oguma E, Kuroki H et al. Clockwise whirlpool sign at color Doppler US: an objective and definite sign of midgut volvulus. Radiology. 1996;199(1):2614.

7. Orzech N, Navarro OM, Langer JC. Is ultrasonography a good screening test for intestinal malrotation? J Pediatr Surg. 2006;41(5):1005-9.

8. Chao HC, Kong MS, Chen JY, Lin SJ, Lin JN. Sonographic features related to volvulus in neonatal intestinal malrotation. $J$ Ultrasound Med. 2000;19(6):371-6.

9. Van Winckel M, Voet D, Robberecht E. "Whirlpool sign": not always associated with volvulus in intestinal malrotation. J Clin Ultrasound.1996;24(7):367-70.

10. Ashley LM, Allen S, Teele RL.A normal sonogram does not exclude malrotation. Pediatr Radiol. 2001;31(5):354-6.

11. Pracros JP, Sann L, Genin G, Tran-Minh VA, Morin de Finfe CH, Foray $P$, et al. Ultrasound diagnosis of midgut volvulus: the "whirlpool" sign. Pediatr Radiol.1992;22(1):18-20.

12. Dufour D, Delaet MH, Dassonville M, Cadranel S, Perlmutter N Midgut malrotation, the reliability of sonographic diagnosis. Pediatr Radiol. 1992;22(1):21-3.

13. Epelman M. The whirlpool sign. Radiology. 2006;240(3):910-1. 